\title{
Excitations and Decays of Rubidium Rydberg States Induced by Blackbody Radiation
}

\begin{abstract}
I.L. Glukhov* And V.V. Chernushinin
Department of Physics, Voronezh State University, 394006, University sq. 1, Voronezh, Russian Federation

The rates of excitations and decays induced by blackbody radiation were calculated in the single-electron Fues' model potential approach for states up to $n=100$ in rubidium $s^{-}, p$ - and $d$-series. On the basis of the calculations, general regularities for maxima rates of blackbody radiation induced decays and excitations were ascertained and a simple polynomial-based approximation formula was proposed for blackbody radiation induced decay and excitation rates of Rydberg states. A temperature parameterization for $T=50-1500 \mathrm{~K}$ was also proposed and respective matrices of coefficients were calculated.
\end{abstract}

PACS numbers:

\section{Introduction}

Blackbody radiation (BBR) is a ubiquitous and inevitable perturbing factor, which affects neutral atoms unless the ambient temperature $T=0 \mathrm{~K}$. It should be taken into account in both laboratory experiments and applied devices engineering. At $T=50-1500 \mathrm{~K}$ the number of thermal photons reaches the maximum in the mid-infrared and far-infrared. These wavelengths correspond to gaps between the Rydberg states, and therefore blackbody radiation affects mainly highly excited states. Thermal photons ionize the Rydberg states [1, 2] and also depopulate them [3] causing the redistribution of electrons by virtue of induced excitations and decays.

Since the pioneer papers $[4,5]$ interlevel transitions induced by $\mathrm{BBR}$ were investigated without separation to BBR-induced decays and BBR-induced excitations. Only the problem of superradiance in the ensembles of the Rydberg atoms requires the account of the decays stimulated by thermal photons; BBR-induced excitations are also the key part of the correct qualitative and quantitative explanation of the selective field ionization processes [2].

This paper provides a systematic investigation of BBR-induced decays and BBR-induced excitations separately in rubidium $s-, p$ - and $d$-series with an account of fine structure of the states. The proposed approximation formulae for BBR-induced decay and excitation rates $\left(P_{n l j}^{\mathrm{d}}\right.$ and $P_{n l j}^{\mathrm{e}}$, respectively) are supplied with a numerical data.

\section{Directly calculated decay and excitation rates}

In one-electron dipole approximation (it is valid because rubidium has a large energy gap between one-

* corresponding author; e-mail: GlukhovOfficial@mail.ru -electron and two-electron excitations; BBR intensity is low at $T=50-1500 \mathrm{~K}$ ), BBR-induced decay and excitation rates for $|n l j\rangle$-state may be calculated in the following way (atomic units are used unless otherwise stated explicitly):

$$
\begin{aligned}
& P_{n l j}^{\mathrm{d}}=\frac{4 \alpha^{3}}{3} \sum_{n^{\prime}, j^{\prime}}^{E_{n^{\prime} l^{\prime} j^{\prime}}<E_{n l j}} l_{>}\left(2 j^{\prime}+1\right)\left\{\begin{array}{ccc}
l & 1 / 2 & j \\
j^{\prime} & 1 & l^{\prime}
\end{array}\right\}^{2} \\
& \times M_{n l j \rightarrow n^{\prime} l^{\prime} j^{\prime}}^{2} \frac{\omega_{n n^{\prime}}^{3}}{\exp \left[\hbar \omega_{n n^{\prime}} /(k T)\right]-1}, \\
& P_{n l j}^{\mathrm{e}}=\frac{4 \alpha^{3}}{3} \sum_{n^{\prime}, j^{\prime}}^{E_{n^{\prime} l^{\prime} j^{\prime}}>E_{n l j}} l_{>}\left(2 j^{\prime}+1\right)\left\{\begin{array}{ccc}
l & 1 / 2 & j \\
j^{\prime} & 1 & l^{\prime}
\end{array}\right\}^{2} \\
& \quad \times M_{n l j \rightarrow n^{\prime} l^{\prime} j^{\prime}}^{2} \frac{\omega_{n n^{\prime}}^{3}}{\exp \left[\hbar \omega_{n n^{\prime}} /(k T)\right]-1},
\end{aligned}
$$

where $\alpha$ is the fine structure constant, $T$ is the temperature of BBR, $k$ - Boltzmann constant, $\omega_{n n^{\prime}}=$ $\left|E_{n l j}-E_{n^{\prime} l^{\prime} j^{\prime}}\right| / \hbar$ :

$$
M_{n l j \rightarrow n^{\prime} l^{\prime} j^{\prime}}=\int_{0}^{\infty} R_{n^{\prime} l^{\prime} j^{\prime}}(r) r R_{n l j}(r) r^{2} \mathrm{~d} r
$$

is a radial matrix element of $|n l j\rangle \rightarrow\left|n^{\prime} l^{\prime} j^{\prime}\right\rangle$ transition with radial wave functions $R_{n l j}(r)$ and $R_{n^{\prime} l^{\prime} j^{\prime}}(r)$, respectively. In this paper, the Fues' model potential radial wavefunctions for the valence electrons in neutral atom [1] were used for the calculations of matrix elements (3):

$$
\begin{aligned}
& R_{n l j}(r)=\frac{2}{\nu^{2}} \sqrt{\frac{(2 \lambda+2)_{n_{\mathrm{r}}}}{n_{\mathrm{r}} ! \Gamma(2 \lambda+2)}} \mathrm{e}^{-r / \nu}\left(\frac{2 r}{\nu}\right)^{\lambda} \\
& \quad{ }_{1} F_{1}\left(-n_{\mathrm{r}} ; 2 \lambda+2 ; \frac{2 r}{\nu}\right),
\end{aligned}
$$

where $\nu$ is the effective principal quantum number $\left(E_{n l j}\right.$ $\left.=-1 /\left(2 \nu^{2}\right)\right) ;{ }_{1} F_{1}(a, c, x)$ is the confluent hypergeometric function, $(a)_{n}=a \cdot(a+1) \cdot \ldots \cdot(a+n-1)$ is the 
Pochhammer symbol, and $n_{\mathrm{r}}$ - the number of nodes in the radial wavefunction. For $s$-states $n_{\mathrm{r}}=n-n_{0}+1$; for $p$ - and $d$-states $n_{\mathrm{r}}=n-n_{0}$, where $n_{0}$ is the principal quantum number of the lowest state for the valence electron in series $(5 s, 5 p, 4 d$ in rubidium). The effective orbital quantum number $\lambda$ is determined from the equation $\nu=\lambda+n_{\mathrm{r}}+1$. Thus, it is necessary to know only one experimental parameter $E_{n l j}$ to construct a radial wavefunction (4). Vast data for $E_{n l j}$ in the Rb states may be found, for example, in NIST database [6].

The Fues model potential wave functions (4) not only provide a good representation of the Rydberg states [7], but also result in an analytical form of matrix element (3):

$$
\begin{aligned}
& M_{n l j \rightarrow n^{\prime} l^{\prime} j^{\prime}}=\frac{1}{4} \frac{\Gamma\left(\lambda+\lambda^{\prime}+4\right)}{\sqrt{\Gamma(2 \lambda+2) \Gamma\left(2 \lambda^{\prime}+2\right)}} \\
& \times \sqrt{\frac{(2 \lambda+2)_{n_{\mathrm{r}}}}{n_{\mathrm{r}} !} \frac{\left(2 \lambda^{\prime}+2\right)_{n_{\mathrm{r}}^{\prime}}}{n_{\mathrm{r}}^{\prime} !}\left(\frac{2 \nu^{\prime}}{\nu+\nu^{\prime}}\right)^{\lambda+2}} \\
& \times\left(\frac{2 \nu}{\nu+\nu^{\prime}}\right)^{\lambda^{\prime}+2} F_{2}\left(\lambda+\lambda^{\prime}+4,-n_{\mathrm{r}},-n_{\mathrm{r}}^{\prime} ;\right. \\
& \left.2 \lambda+2,2 \lambda^{\prime}+2 ; 2 \nu^{\prime} /\left(\nu+\nu^{\prime}\right), 2 \nu^{\prime}\left(\nu+\nu^{\prime}\right)\right),
\end{aligned}
$$

where $F_{2}\left(a, b_{1}, b_{2} ; c_{1}, c_{2} ; x_{1}, x_{2}\right)$ is a generalized hypergeometric function with five parameters and two arguments.

Matrix elements (5) were calculated for necessary transitions with $n$ up to 200 and stored for quick implementation in (1) and (2) at different temperatures. The total depopulation rates at $T=300 \mathrm{~K}$ are in good agreement with previous results [4]. The calculations demonstrated that photoexcitation and photodecay rates have distinct maxima. The state with the maximal rate in a series changes with temperature, because the maximum of the spectral radiant energy density $u_{\omega}(T)$ is shifted with changing temperature $(\approx 2.82 k T)$. That is, the maximum of energy density coincides with different transitions at different temperatures. As the transitions to the nearest states (with energy gaps $\approx 1 / \nu^{3}$ ) give the main tribute to rates, the state follows the equation:

$$
C_{\mathrm{m}}=\frac{100}{\nu T^{1 / 3}},
$$

where $C_{\mathrm{m}}$ is the constant of the maximum for the series, and $T$ is in kelvin. The values for $C_{\mathrm{m}}$ were found in $s^{-}, p-, d$-series of rubidium for BBR-induced decay, exci-

\section{TABLE}

The constants of the maximum in $\mathrm{Rb}$ (rubidium).

\begin{tabular}{l|c|c|c}
\hline \multicolumn{1}{c|}{ Series } & $s$ & $p$ & $d$ \\
\hline decay & 1.8 & 1.65 & 1.7 \\
excitation & 1.9 & 2.05 & 2.0 \\
total & 1.85 & 1.8 & 1.8
\end{tabular}

tation and total depopulation (sum of decay and excitation) rates. The maximum constants are presented in the table, which demonstrates a quantitative difference between two qualitatively different photoinduced processes.

\section{Approximation}

The number of the Rydberg states in each investigated series of rubidium is infinitely large; therefore it is not efficient to represent direct calculation results for BBR-induced decay and excitation rates in the form of vast tables. The more proper and practical approach is to construct a simple approximation.

The transitions to the closest in energy states $(\mid n-$ $n^{\prime} \mid \sim 1$ ) provide a main tribute to the rates of both photoinduced decay and photoinduced excitation processes. Therefore the first step in an approximation construction is to replace $\omega_{n n^{\prime}}$ with interlevel energy gap asymptotic $1 / \nu^{3}$ and matrix elements with $\nu^{2}$ asymptotic. The second step of the approximation is the account of other transitions $\left(\left|n-n^{\prime}\right|>1\right)$ by means of introducing the inverse powers of $\nu$ in a polynomial form in the terms of the dimensionless parameter $x=100 /\left(\nu T^{1 / 3}\right)$ :

$$
P_{n l j}^{\mathrm{d}(\mathrm{e})}=\frac{a_{0}^{\mathrm{d}(\mathrm{e})}\left(1+a_{1}^{\mathrm{d}(\mathrm{e})} x+a_{2}^{\mathrm{d}(\mathrm{e})} x^{2}+a_{3}^{\mathrm{d}(\mathrm{e})} x^{3}\right)}{\tilde{\nu}^{5}\left[\exp \left(0.315792 x^{3}\right)-1\right]}
$$

with $\tilde{\nu}=\frac{\nu}{100}$. Equation (6) requires the temperature of $\mathrm{BBR}$ in kelvin and provides decay and excitation rates in inverse seconds $(1 / \mathrm{s}) . a_{0}^{\mathrm{d}(\mathrm{e})}-$ coefficients fix correct asymptotic behavior for high- $n$ Rydberg states in the series and do not depend on the temperature. The terms with the coefficients $a_{1}^{\mathrm{d}(\mathrm{e})}, a_{2}^{\mathrm{d}(\mathrm{e})}, a_{3}^{\mathrm{d}(\mathrm{e})}$ reflect the behavior of the approximation near the maximum and on the shallow slope. The coefficients $a_{1}^{\mathrm{d}(\mathrm{e})}, a_{2}^{\mathrm{d}(\mathrm{e})}, a_{3}^{\mathrm{d}(\mathrm{e})}$ slightly depend on the temperature and may be parameterized in the following way

$$
a_{i}^{\mathrm{d}(\mathrm{e})}=\sum_{k=0}^{2} b_{i k}^{\mathrm{d}(\mathrm{e})}\left(\frac{T}{100}\right)^{-k}, \quad i=0,1,2,3 .
$$

So, the knowledge of $a_{0}^{\mathrm{d}(\mathrm{e})}-$ coefficient and $b_{i k}^{\mathrm{d}(\mathrm{e})}-$ matrix provides enough information on BBR-induced decay (excitation) rates in a given series.

\section{Results}

Direct calculations of BBR-induced decays (1) and excitations (2) in each series for $n$ up to 100 gave a basis for fitting $a_{i}^{\mathrm{d}(\mathrm{e})}$ - coefficients in Eq. (6) as the first step. Then the sets of $a_{i}^{\mathrm{d}(\mathrm{e})}$ - coefficients at different temperatures were used for fitting $b_{i k}^{\mathrm{d}(\mathrm{e})}-$ coefficients. The obtained data demonstrates a good approximation of direct calculations (see Fig. 1).

$$
\begin{gathered}
\text { 4.1. s-states } \\
a_{0}^{\mathrm{d}}=1.104, \quad b_{i k}^{\mathrm{d}}=\left(\begin{array}{ccc}
-0.2391 & 0.0595 & 0.0314 \\
-0.1705 & 0.1821 & -0.0844 \\
0.1262 & -0.0332 & 0.0225
\end{array}\right),
\end{gathered}
$$




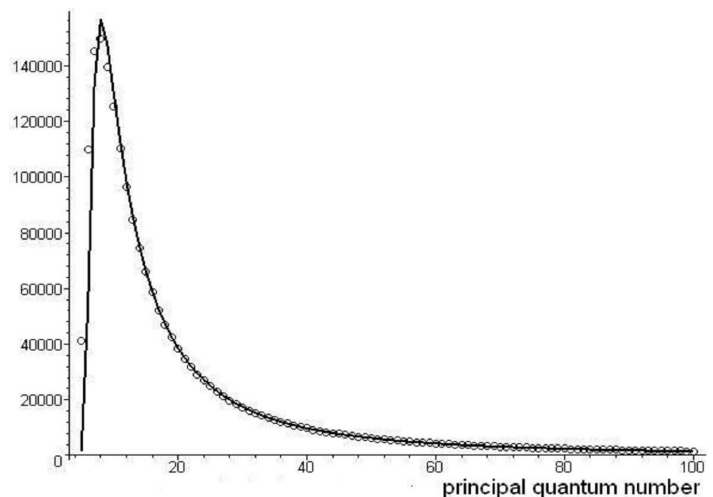

Fig. 1. Excitation rates (1/s) of Rydberg states in $d_{3 / 2}$-series at $600 \mathrm{~K}$; circles - direct calculations, full line - approximation.

$$
a_{0}^{\mathrm{e}}=0.9342, \quad b_{i k}^{\mathrm{e}}=\left(\begin{array}{ccc}
0.1310 & 0.1185 & -0.0443 \\
-0.7012 & 0.0028 & 0.0193 \\
0.4646 & -0.0751 & 0.0127
\end{array}\right) \text {. }
$$$$
\text { 4.2. } p_{1 / 2} \text {-states }
$$

$$
\begin{aligned}
& a_{0}^{\mathrm{d}}=1.4885, \quad b_{i k}^{\mathrm{d}}=\left(\begin{array}{ccc}
-0.5184 & 0.1206 & -0.0406 \\
0.0644 & -0.0680 & 0.0218 \\
0.0666 & 0.0134 & 0.0037
\end{array}\right), \\
& a_{0}^{\mathrm{e}}=0.5732, \quad b_{i k}^{\mathrm{e}}=\left(\begin{array}{ccc}
0.1968 & 0.1727 & -0.0608 \\
-0.6840 & -0.1673 & 0.0676 \\
0.4136 & 0.0714 & -0.0306
\end{array}\right) .
\end{aligned}
$$

$$
\text { 4.3. } p_{3 / 2} \text {-states }
$$

$$
\begin{aligned}
& a_{0}^{\mathrm{d}}=1.5414, \quad b_{i k}^{\mathrm{d}}=\left(\begin{array}{ccc}
-0.5260 & 0.0325 & -0.0047 \\
0.0690 & 0.0160 & -0.0076 \\
0.0681 & -0.0194 & 0.0061
\end{array}\right), \\
& a_{0}^{\mathrm{e}}=0.5505, \quad b_{i k}^{\mathrm{e}}=\left(\begin{array}{ccc}
0.0485 & 0.2301 & -0.0706 \\
-0.4515 & -0.4157 & 0.1581 \\
0.3336 & 0.1956 & -0.0786
\end{array}\right) .
\end{aligned}
$$

$$
\text { 4.4. } d_{3 / 2} \text {-states }
$$

$$
a_{0}^{\mathrm{d}}=1.1936, \quad b_{i k}^{\mathrm{d}}=\left(\begin{array}{ccc}
-0.9873 & 0.6452 & -0.2224 \\
0.4247 & -0.5073 & 0.1756 \\
-0.0397 & 0.1441 & -0.0486
\end{array}\right) \text {, }
$$

$$
\begin{array}{rr}
a_{0}^{\mathrm{e}}=0.8134, \quad b_{i k}^{\mathrm{e}}= & \left(\begin{array}{ccc}
-0.1647 & 0.3949 & -0.1385 \\
-0.0770 & -0.6981 & 0.2396 \\
0.1398 & 0.2623 & -0.0916
\end{array}\right) . \\
\text { 4.5. } d_{5 / 2} \text {-states } \\
a_{0}^{\mathrm{d}}=1.1858, \quad b_{i k}^{\mathrm{d}}=\left(\begin{array}{ccc}
-1.0085 & 0.6949 & 0.2437 \\
0.4394 & -0.5832 & 0.2116 \\
-0.0436 & 0.1777 & -0.0656
\end{array}\right), \\
a_{0}^{\mathrm{e}}=0.8215, \quad b_{i k}^{\mathrm{e}}=\left(\begin{array}{ccc}
-0.0865 & 0.2614 & -0.0519 \\
-0.2152 & -0.4875 & 0.1002 \\
0.1931 & 0.1732 & -0.0357
\end{array}\right) .
\end{array}
$$

\section{Conclusion}

The Fues model potential was successfully employed for the calculation of BBR-induced decay and excitation rates in $\mathrm{Rb} s-, p$ - and $d$ - Rydberg states. The obtained results were approximated with a simple analytical formula (6) and a temperature parameterization (7). They provide a correct reflection of maximal rates, and deviations in high- $n$ asymptotic area not more than $2 \%$.

\section{Acknowledgments}

This work was supported by Russian Foundation for the Basic Researches (grant number 07-02-00279a) and by the President of Russian Federation (grant number MK 251.2008.2).

\section{References}

[1] I.L. Glukhov, V.D. Ovsiannikov, J. Phys. B 42, 075001 (2009).

[2] I.I. Beterov, D.B. Tretyakov, I.I. Ryabtsev, V.M. Entin, A. Ekers, N.N. Bezuglov, New J. Phys. 11, 013052 (2009).

[3] I.I. Beterov, I.I. Ryabtsev, D.B. Tretyakov, V.M. Entin, Phys. Rev. A 79, 052504 (2009).

[4] J.W. Farley, W.H. Wing, Phys. Rev. A 23, 2397 (1981).

[5] C.E. Theodosio, Phys. Rev. A 30, 2881 (1984).

[6] http://physics.nist.gov/asd3 .

[7] A.A. Kamenski, V.D. Ovsiannikov, J. Phys. B 39, 2247 (2006) 